\title{
Membrane charge oscillations during ultrasonic neuromodulation by intramembrane cavitation
}

\author{
Thomas Tarnaud, Wout Joseph, Ruben Schoeters, Luc Martens, Emmeric Tanghe
}

\begin{abstract}
Objective: To investigate the importance of membrane charge oscillations and redistribution in multicompartmental ultrasonic neuromodulation (UNMOD) intramembrane cavitation models. Methods: The Neuronal Intramembrane Cavitation Excitation (NICE) model and multiScale Optimized model of Neuronal Intramembrane Cavitation (SONIC) of UNMOD are compared for a nanoscale multicompartmental and point neuron approximation of the bilayer sonophore and surrounding proteins. The temporal dynamics of charge oscillations and their effect on the resulting voltage oscillations are investigated by fourier series analysis. Results: Comparison of excitation thresholds and neuronal response between nanoscale multi-compartmental and point models, implemented in the SONIC and NICE framework, demonstrates that the explicit modeling of fast spatial charge redistribution is critical for an accurate multi-compartmental UNMOD-model. Furthermore, the importance of modeling partial protein coverage is quantified by the excitability thresholds. Subsequently, we establish by fourier analysis that these charge oscillations are slowly changing in time. Conclusion: Fast charge redistribution significantly alters neuronal excitability in a multi-compartmental nanoscale UNMOD-model. Also the mutual exclusivity between protein and sonophore coverage should be taken into account, when simulating the dependency of neuronal excitability on coverage fractions. Charge oscillations are periodic and their fourier components change on a slow timescale. Furthermore, the resulting voltage oscillations decrease in energy with overtone number, implying that an extension of the existing multiscale model (SONIC) to multi-compartmental neurons is possible by taking into account a limited number of fourier components. Significance: First steps are taken towards a morphologically realistic and computationally efficient UNMOD-model, improving our understanding of the underlying ultrasonic neuromodulation mechanisms.
\end{abstract}

Index Terms-Ultrasonic neuromodulation, intramembrane cavitation, bilayer sonophore, charge oscillations, computational neuroscience

This work was carried out using the Supercomputer Infrastructure (STEVIN) at Ghent University, funded by Ghent University, the Flemish Supercomputer Center (VSC), the Hercules Foundation and the Flemish Government department EWI.

This research was funded by the FWO-project G046816N. T. Tarnaud is a PhD Fellow of the FWO-V (SB) (Research Foundation Flanders, Belgium). $\mathrm{R}$. Schoeters is a PhD Fellow of the FWO-V (Research Foundation Flanders, Belgium).

T. Tarnaud, W. Joseph, R. Schoeters, L. Martens, and E. Tanghe are with the Department of Information Technology (INTEC-WAVES/IMEC), Ghent University/IMEC, Technologypark 126, 9052 Zwijnaarde, Belgium. E-mail: thomas.tarnaud@ugent.be

Copyright (c) 2017 IEEE. Personal use of this material is permitted. However, permission to use this material for any other purposes must be obtained from the IEEE by sending an email to pubs-permissions@ieee.org.

\section{INTRODUCTION}

$\mathbf{U}$ LTRASONIC neuromodulation (UNMOD) has gained significant attention in the last decade, because of its capability to modulate brain activity reversibly, non-invasively, selectively, and with high spatial resolution (transversal fullwidth at half maximum in the order of millimeters with a single element focused transducer) [1] -[15]. Furthermore, a good safety profile has been reported [16], [17] and there is no requirement to genetically alter target neurons (cfr. optogenetics [18]-[21] and wireless magnetothermal brain stimulation [22]). Another advantage of UNMOD is that, although a significant difference is expected between the insonicated white and grey matter neuronal response, ultrasonic pressure and velocity fields exhibit a lower dependency on anatomical properties, e.g., gyral curvature, tissue anisotropy... [23] (cfr. inter-subject variability in transcranial magnetic stimulation and direct current stimulation [24], [25]).

Unfortunately, the underlying mechanism of ultrasonic neuromodulation is not well understood, precluding detailed optimization of ultrasonic waveforms and transducers for the application of neuromodulation. In this study, we concentrate on the bilayer sonophore model (BLS) of intramembrane cavitation [26]-[28], which has been able to predict several features of low-intensity ultrasonic neuromodulation (e.g., insonication induced action potentials, threshold-frequency plots, interplay of membrane dynamics and duty cycle (cell-type selectivity), dependency of the success rate on intensity, pulse duration and frequency...). We will refer to the Plaksin-Shoham-Kimmel (PSK) model, as the mathematical/conceptual framework in which bilayer sonophores are the main elements for the conversion of ultrasonic to electrical energy (action potentials) in neurons.

However, the extension of the PSK-model to multicompartmental neurons has been hindered by the computational stiffness of the model, caused by the simultaneous importance of the very short timescale of membrane oscillations set by the ultrasonic frequency (microseconds), the intermediate timescale of membrane dynamics (milliseconds in action potentials), and the relatively long timescale of spike-amplitude and spike-frequency adaptation (seconds). In a multi-compartmental model, computational stiffness is significantly increased due to strong axial currents between neighbouring compartments caused by the large membrane voltage oscillations that are seen in the PSK-model [29]. As a result, very stringent tolerances and maximal time steps have to be applied upon the used differential solvers, resulting in exorbitant simulation times for parameter sweeps 
or longer insonication durations. Despite these difficulties, a multi-compartmental model of ultrasonic neuromodulation by intramembrane cavitation is important in order to further improve understanding of the underlying mechanism of UNMOD.

In literature, two attempts have been made to reduce the computational stiffness of the PSK-model. First, in the Neuronal Intramembrane Cavitation Excitation (NICE) model, the authors of the PSK-model (Plaksin, Shoham, Kimmel) proposed the decoupling of the mechanical Rayleigh-Plesset equation and the Hodgkin-Huxley equation, by introducing an update time for the electrostatic Coulomb interaction between the two leaflets 1 while making use of the pseudo-periodicity of the sonophore dynamics. Second, Lemaire et al. (2019) introduced the multiScale Optimized Neuronal model of Intramembrane Cavitation (SONIC), in which a three orders of magnitude computational speed-up is achieved [30]. Here, the stiffness of the PSK-model is reduced by pretabulation of effective rate constants and effective potential values, removing the smallest timescale that is determined by the ultrasonic period from the computational implementation, while maintaining accurate results for point neurons. Furthermore, further reductions in simulation time are obtained in Lemaire et al. by introducing a Lennard-Jones approximation of the molecular pressure between the leaflets of the bilipid layer and by charge recasting of the modified Hodgkin-Huxley equations. Interestingly, in Lemaire et al. a first attempt is also made to extend the SONIC-framework towards multicompartmental models: a two-compartment nanoscale model of the bilayer sonophore and surrounding proteins is introduced. Subsequently, it is shown in [30] that the twocompartment nanoscale SONIC-model predicts much lower excitation thresholds as compared to the point-neuron NICE implementation, for partial sonophore coverage.

In this study, we will use the two-compartment nanoscale model of the BLS and surrounding proteins as our starting point to investigate the possibility of computational efficient implementations of multicompartmental UNMOD models. First, we demonstrate that a single-compartment (point neuron) implementation of the nanoscale sonophore and surrounding proteins is accurate, when modeling UNMOD by intramembrane cavitation. As a result, this nanoscale model is useful as a benchmark for computationally efficient and accurate multicompartmental PSK-models, because the solution is known. Second, we demonstrate the importance of accounting for charge oscillations and redistribution between compartments in a multi-compartmental model of UNMOD by intramembrane cavitation. We argue that the discrepancy of excitation thresholds, observed in Lemaire et al. [30] between the twocompartment SONIC model and single-compartment NICEmodel, is caused by this fast oscillation and redistribution of the membrane charge between the sonophore and the protein

\footnotetext{
${ }^{1}$ In literature, the terms 'PSK-model' and 'NICE-model' are used interchangeably. For convenience, we will use the former to refer to the conceptual/mathematical bilayer sonophore model for neuronal insonication, while the latter will be used in the context of the specific implementation of the PSK-model in Plaksin et al. (2014) (in particular, with an update time for the Coulomb pressure) [27]
}

islands. Because the multiscale approximation of the SONICmodel implicitly assumes a slow (millisecond) timescale for the membrane charge, fast (ultrasound timescale) charge redistribution and oscillations between different compartments are not taken into account. These microsecond scale pseudoperiodic charge oscillations impact neuronal excitability by two non-linear mechanisms: the ion channel gating and the electrostatic interaction between both charged lipid leaflets. Third, until now, coverage by transmembrane proteins has been considered independent of sonophore coverage in intramembrane cavitation models. We illustrate that accounting for the mutual exclusivity between protein and sonophore coverage, results in excitability curves that are not monotonously decreasing with sonophore coverage fraction. Instead, an intermediate value of sonophore coverage will be optimal for ultrasonic neuromodulation by intramembrane cavitation. To this end, two models with mutual exclusivity between protein and sonophore coverage (i.e., partial protein coverage: no voltage-gated protein channels in the sonophore compartment) are compared with the biologically unrealistic model case with full protein coverage (i.e., protein channels in the sonophore). Fourth, first steps are taken for the design of a multi-scale optimized multi-compartmental model that accounts for fast charge oscillations. Here, we show that, while the membrane charge is oscillating at the same microsecond timescale as the membrane potential in a multi-compartmental model, the fourier components of these oscillations are changing on the slow timescale of the membrane gates. Furthermore, most of the signal energy of the resulting voltage oscillation is found in the lowest overtone numbers. These results imply that a multi-compartmental extension of the SONIC-framework is possible, by including a set of equations to describe the most important fourier spectrum components of the membrane charge oscillations.

\section{Methods}

First, we briefly discuss the bilayer sonophore model [26] and the PSK-model [27], [28] in subsections II-A and II-B] respectively. Subsequently, a discussion of the point neuron and nanoscale multicompartmental model are given in subsection II-C Second, the implementation of the PSKmodel is discussed: the NICE [27], [28] and the SONIC [30] implementation in subsection II-D and II-E, respectively. A schematical overview of the used methodology and PSKmodels is shown in Fig. S1 in the supplementary information.

The definition and values of the general model constants are the same as in our previous computational study on ultrasonic neuromodulation of the subthalamic nucleus [31] (summarized in Table SI in the supplementary information). A summary of the definition and units of the used model variables and functions is given in Table SII.

\section{A. Bilayer sonophore (BLS) model}

The bilayer sonophore model (BLS; [26]) describes the oscillation of intramembrane air pockets by a modified Rayleigh- 
TABLE I: Summary of Membrane Current Model Parameters

\begin{tabular}{|c|c|c|}
\hline Membrane current & $g\left[\mathrm{mS} / \mathrm{cm}^{2}\right]$ & $V[\mathrm{mV}]$ \\
\hline$I_{\mathrm{Na}}$ & 56 & 50 \\
$I_{\mathrm{K}}$ & 6 & -90 \\
$I_{\mathrm{M}}$ & 0.075 & -90 \\
$I_{1}$ & 0.0205 & -70.3 \\
\hline
\end{tabular}

Plesset equation of bubble dynamics:

$$
\begin{gathered}
\frac{\mathrm{d}^{2} Z}{\mathrm{~d} t^{2}}+\frac{3}{2 R(Z)}\left(\frac{\mathrm{d} Z}{\mathrm{~d} t}\right)^{2}=\frac{1}{\rho_{\mathrm{l}}|R(Z)|}\left[P_{\mathrm{in}}+P_{\mathrm{M}}+P_{\mathrm{ec}}-P_{0}+\right. \\
\left.P_{\mathrm{A}} \sin (\omega t)-P_{\mathrm{S}}(Z)-\frac{4}{|R(Z)|} \frac{\mathrm{d} Z}{\mathrm{~d} t}\left(\frac{3 \delta_{0} \mu_{\mathrm{s}}}{|R(Z)|}+\mu_{\mathrm{l}}\right)\right] .
\end{gathered}
$$

Here, the radius of curvature is calculated by $R(Z)=\left(a_{\mathrm{BLS}}^{2}+\right.$ $\left.Z^{2}\right) /(2 Z)$, with $Z$ the maximal deflection of the inner and outer leaflets of the phospholipid layer. The applied pressure $P_{\mathrm{A}}$ oscillates at angular frequency $\omega=2 \pi f_{\mathrm{US}}$ (phase is set to zero by convention).

Membrane oscillations are caused by the balance of different pressure terms. The gas pressure is described by the ideal gas law:

$$
P_{\text {in }}=\frac{n_{\mathrm{a}} R_{\mathrm{g}} T}{V_{\mathrm{a}}(Z)} .
$$

The intramembrane air concentrantion is calculated by a diffusion equation:

$$
\frac{\mathrm{d} n_{\mathrm{a}}}{\mathrm{d} t}=\frac{2 S(Z) D_{\mathrm{a}}}{\xi}\left[C_{\mathrm{a}}-P_{\mathrm{in}} / k_{\mathrm{a}}\right] .
$$

$S(Z)=\pi\left(a_{\mathrm{BLS}}^{2}+Z^{2}\right)$ is the area of a single leaflet and $V_{\mathrm{a}}(Z)=\pi a_{\mathrm{BLS}}^{2} \Delta\left[1+Z /(3 \Delta)\left(\frac{Z^{2}}{a_{\mathrm{BLS}}^{2}}+3\right)\right]$ is the intramembrane volume.

The molecular pressure $P_{\mathrm{M}}$ requires the evaluation of the integral:

$$
P_{\mathrm{M}}=A_{\mathrm{r}} \frac{2 \pi}{S(Z)} \int_{0}^{a} r \mathrm{~d} r\left[\left(\frac{\Delta^{*}}{2 z(r)+\Delta}\right)^{x}-\left(\frac{\Delta^{*}}{2 z(r)+\Delta}\right)^{y}\right],
$$

with the local leaflet displacement:

$$
z(r)=\operatorname{sign}(R(Z)) \sqrt{R(Z)^{2}-r^{2}}-R(Z)+Z .
$$

The electrostatic (Coulomb) pressure $P_{\mathrm{ec}}$ and the pressure caused by membrane tension $P_{\mathrm{S}}$ are given by:

$$
\begin{gathered}
P_{\mathrm{ec}}=-\frac{\pi a^{2}}{S(Z)} \frac{Q_{\mathrm{m}}^{2}}{2 \epsilon_{\mathrm{r}} \epsilon_{0}}, \\
P_{\mathrm{S}}(Z)=\frac{2 k_{\mathrm{s}} Z^{3}}{a_{\mathrm{BLS}}^{2}\left(a_{\mathrm{BLS}}^{2}+Z^{2}\right)} .
\end{gathered}
$$

Here, $Q_{\mathrm{m}}=C_{\mathrm{m}} V$ is the membrane charge.

We initialize the leaflet position at $t=0 \mathrm{~ms}$ with $Z=0 \mathrm{~nm}$ and $\frac{\mathrm{d} Z}{\mathrm{~d} t}=0 \mathrm{~nm} / \mathrm{s}$. Because the Rayleigh-Plesset equation fails at these conditions, a quasistatic approximation is used until $Z \geq Z_{\mathrm{QS}}\left(Z_{\mathrm{QS}}=0.001 \mathrm{~nm}\right)$ (cfr. supplementary information in [26]):

$$
P_{\mathrm{in}}^{\mathrm{QS}}+P_{\mathrm{M}}+P_{\mathrm{A}} \sin (\omega t)+P_{\mathrm{ec}}-P_{0}=0 .
$$

For the internal gas pressure $P_{\text {in }}^{\mathrm{QS}}$ a quasi-static approximation is used:

$$
P_{\mathrm{in}}^{\mathrm{QS}}=P_{0} \frac{\pi a^{2} \Delta}{V_{\mathrm{a}}(Z)^{\kappa}} .
$$

TABLE II: Membrane Protein Dynamics

\begin{tabular}{|c|c|c|}
\hline$X$ & $\alpha_{X}[1 / \mathrm{ms}]$ & $\beta_{X}[1 / \mathrm{ms}]$ \\
\hline$m$ & $\frac{-0.32\left(V-V_{T}-13\right)}{\exp \left[-\left(V-V_{T}-13\right) / 4\right]-1}$ & $\beta_{m}=0.28 \frac{\left(V-V_{T}-40\right)}{\exp \left[\left(V-V_{T}-40\right) / 5\right]-1}$ \\
$h$ & $0.128 \exp \left[-\left(V-V_{T}-17\right) / 18\right]$ & $\frac{4}{1+\exp \left[-\left(V-V_{T}-40\right) / 5\right]}$ \\
$n$ & $-0.032 \frac{V-V_{T}-15}{\exp \left[-\left(V-V_{T}-15\right) / 5\right]-1}$ & $0.5 \exp \left[-\left(V-V_{T}-10\right) / 40\right]$ \\
\hline & $X_{\infty}[-]$ & $\tau_{X}[\mathrm{~ms}]$ \\
\hline$p$ & $\frac{1}{1+\exp [-(V+35) / 10]}$ & $\frac{608}{3.3 \exp [(V+35) / 20]+\exp [-(V+35) / 20]}$ \\
\hline
\end{tabular}

For a small volume of air in the intraleaflet cavity, the polytropic constant can be approximated by $\kappa=1$.

\section{B. UNMOD by intramembrane cavitation: PSK-model}

In the PSK-model, developed by Plaksin, Shoham and Kimmel, ultrasonic neuromodulation caused by intramembrane cavitation is assumed to be the result of the corresponding oscillations in membrane capacitance [27], [28]:

$$
C_{\mathrm{m}} \frac{\mathrm{d} V}{\mathrm{~d} t}+V \frac{\mathrm{d} C_{\mathrm{m}}}{\mathrm{d} t}=-I_{m}
$$

The dependency of the sonophore membrane capacitance $C_{\mathrm{BLS}}$ on the antiphase apex deflection $Z$ is calculated by:

$C_{\mathrm{BLS}}(Z)=\frac{C_{\mathrm{m} 0} \Delta}{a_{\mathrm{BLS}}^{2}}\left(Z+\frac{a_{\mathrm{BLS}}^{2}-Z^{2}-Z \Delta}{2 Z} \ln \left(\frac{2 Z+\Delta}{\Delta}\right)\right)$.

In this paper, we use the membrane dynamics of a cortical regular spiking neuron [32], as also used in previous computational studies of ultrasonic neuromodulation [27], [28], [30], [33]:

$$
\begin{array}{r}
I_{\mathrm{m}}=g_{\mathrm{Na}} m^{3} h\left(V-V_{\mathrm{Na}}\right)+g_{\mathrm{K}} n^{4}\left(V-V_{\mathrm{K}}\right)+\ldots \\
g_{\mathrm{M}} p\left(V-V_{\mathrm{K}}\right)+g_{\mathrm{l}}\left(V-E_{\mathrm{l}}\right)
\end{array}
$$

The conductances $\left(g_{\mathrm{Na}}, g_{\mathrm{K}}, g_{\mathrm{M}}, g_{1}\right)$ and Nernst-potentials $\left(V_{\mathrm{Na}}, V_{\mathrm{K}}, V_{\mathrm{M}}=V_{\mathrm{K}}, V_{1}\right)$ of the sodium, delayed-rectifier potassium, slowly non-inactivating potassium and leak currents are tabulated in Table I]

The dynamics of the gate parameters is given by [32]:

$$
\frac{\mathrm{d} X}{\mathrm{~d} t}=\frac{X_{\infty}-X}{\tau_{X}} .
$$

Here, $X_{\infty}=\alpha_{X} /\left(\alpha_{X}+\beta_{X}\right)$ and $\tau_{X}=1 /\left(\alpha_{X}+\beta_{X}\right) . X$ represents $m, h, n$ or $p$ : formulas are given in Table [I]

\section{Point neuron and nanoscale multi-compartmental model}

In the case of partial sonophore coverage (coverage ratio $f_{\mathrm{BLS}}=a_{\mathrm{BLS}}^{2} / b^{2} ; b$ is the radius of the modeled cylindrical membrane patch including protein islands) two compartments can be distinguished [30] (Fig. S1): the bilayer sonophore (BLS) with variable oscillating capacitance $C_{\mathrm{BLS}}$ and the surrounding protein islands $(\mathrm{P})$ with fixed capacitance $C_{\mathrm{P}}=C_{\mathrm{m} 0}$.

In this nanoscale multicompartmental model [30], the two compartments are coupled by the resistance:

$$
R_{\mathrm{BLS}, \mathrm{P}}=\int_{\frac{a_{\mathrm{BLS}}}{2}}^{\frac{a_{\mathrm{BLS}}+b}{2}} \frac{\rho_{i}}{2 \pi r d_{\mathrm{eff}}} \mathrm{d} r=\frac{\rho_{i}}{2 \pi d_{\mathrm{eff}}} \ln \frac{a_{\mathrm{BLS}}+b}{a_{\mathrm{BLS}}}
$$


As observed in Lemaire et al. [30], although the resistance depends on the arbitrary parameter $d_{\text {eff }}$, a decrease to a value several orders of magnitude lower than the nanometer range is required to obtain desynchronized activity between the BLS and the protein islands.

The system of equations for both compartments is:

$$
\begin{gathered}
C_{\mathrm{BLS}} \frac{\mathrm{d} V}{\mathrm{~d} t}+V \frac{\mathrm{d} C_{\mathrm{BLS}}}{\mathrm{d} t}=\frac{1}{\pi a_{\mathrm{BLS}}^{2}} \frac{V_{\mathrm{P}}-V_{\mathrm{BLS}}}{R_{\mathrm{BLS}, \mathrm{P}}}-I_{\mathrm{m}, \mathrm{BLS}} \\
C_{\mathrm{P}} \frac{\mathrm{d} V}{\mathrm{~d} t}=\frac{1}{\pi\left(b^{2}-a_{\mathrm{BLS}}^{2}\right)} \frac{V_{\mathrm{BLS}}-V_{\mathrm{P}}}{R_{\mathrm{BLS}, \mathrm{P}}}-I_{\mathrm{m}, \mathrm{P}}
\end{gathered}
$$

For the model case with partial protein coverage, no voltagegated protein channels contribute to the sonophore membrane current $I_{\mathrm{m}, \mathrm{BLS}}$.

In contrast, a point neuron (single compartment) approach to model partial sonophore coverage [28] is to use the following relation between the average membrane capacitance $C_{\mathrm{m}}$ and the bilayer sonophore capacitance $C_{\mathrm{BLS}}$ :

$$
C_{\mathrm{m}}=f_{\mathrm{BLS}} C_{\mathrm{BLS}}+\left(1-f_{\mathrm{BLS}}\right) C_{\mathrm{P}}
$$

Here, the modeled membrane patch (protein islands and bilayer sonophore) are equipotential and local membrane charge can be calculated in the NICE-implementation (subsection II-D by $q(r, t)=C_{\mathrm{m} 0} V \frac{\Delta}{2 z(r, t)+\Delta}$.

\section{Neuronal intramembrane cavitation excitation model}

In the neuronal intramembrane cavitation excitation (NICE) implementation, introduced by Plaksin et al. [27], the stiffness of the complete set of equations of the PSK-model is reduced, by separation of the mechanical problem (1) and the neurodynamical problem $(\sqrt{10})$ or $(15)-(16)$ for the point and nanoscale multicompartmental model, respectively).

The main assumption of the NICE-implementation is that the dynamics of the leaflet do not depend strongly on changes of the membrane charge, that occur within the update time $T_{\text {up }}$. In this study, the membrane charge $Q_{\mathrm{m}}$ in the Coulomb pressure (6) is updated every $T_{\text {up }}=25 \mu \mathrm{s}$. The RayleighPlesset equation (1) is then integrated separately by an ode113solver (variable step variable order (VSVO, order between 1 and 13) solver with Adams-Bashfort-Moulton predictorcorrector pairs) in Matlab ${ }^{\circledR}$ [34], [35] (relative tolerance is 0.0001 , absolute tolerances on the displacement $Z$, leaflet velocity $\mathrm{d} Z / \mathrm{d} t$ and gas content $n_{\mathrm{a}}$ are set to $0.1 \mathrm{pm}$, $1 \mathrm{fm} / \mu \mathrm{s}, 10^{-24}$ mole, respectively, maximal time step $\mathrm{d} t=$ $\left.0.025 / f_{\mathrm{US}}\right)$, until periodicity is detected by thresholding the autocorrelation of a moving-window over the displacement $Z$ and intraleaflet gas content $n_{\mathrm{a}}$.

Subsequently the Hodgkin-Huxley equation (10) is integrated over $T_{\text {up }}$ with an ode113-solver for the point neuron model (relative tolerance 0.001, absolute tolerance $1 \mu \mathrm{V}$ (voltage) and 0.001 (gates), maximal step $\mathrm{d} t=0.025 / f_{\mathrm{US}}$ ). For the nanoscale multi-compartmental model an ode15s-

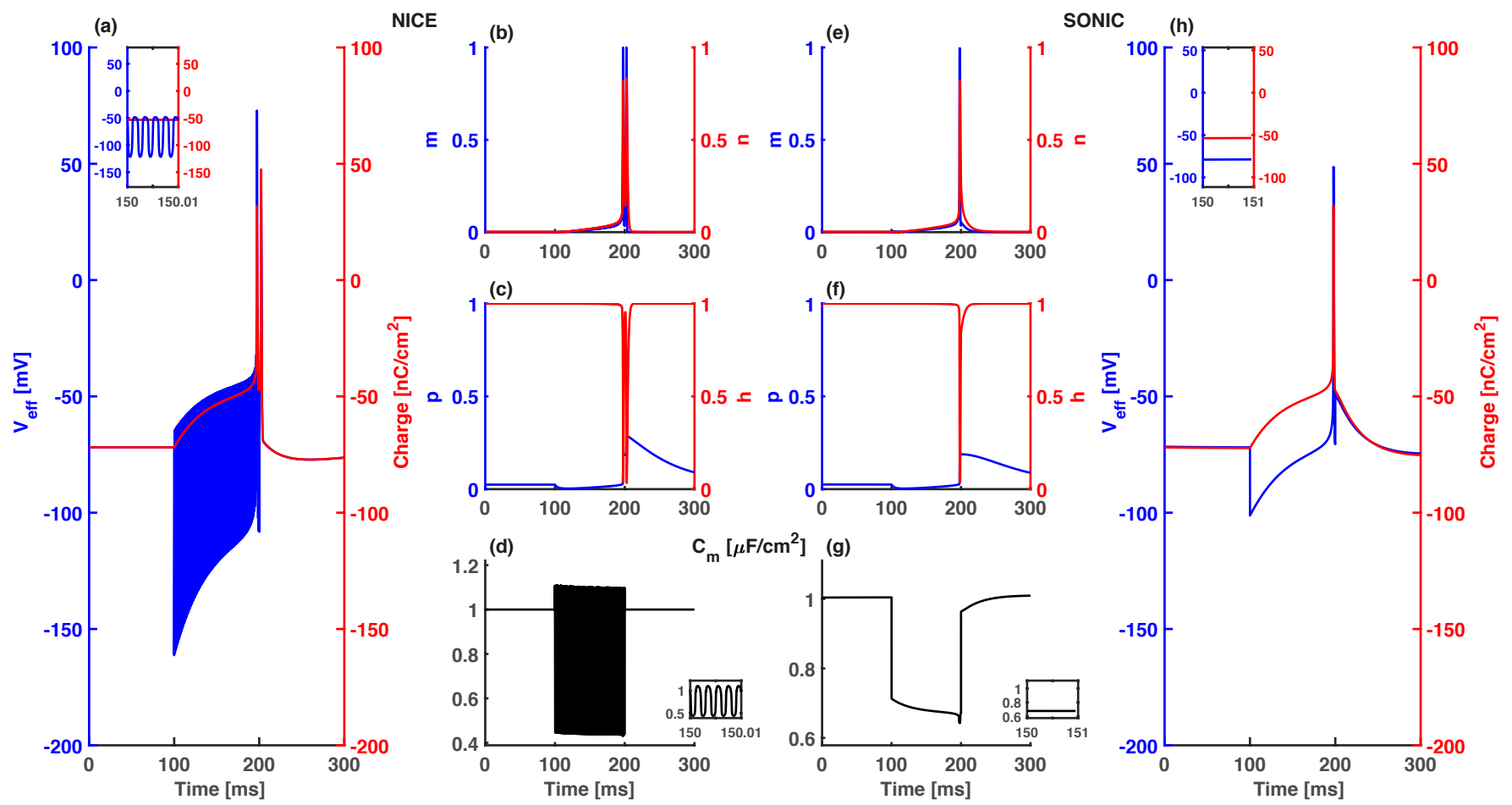

Fig. 1: Insonication of regular spiking point neuron (continuous wave ultrasound, pulse onset and pulse duration set to $100 \mathrm{~ms}$, $\left.P_{\mathrm{US}}=100 \mathrm{kPa}\left(I_{\mathrm{sp}}=321 \mathrm{~mW} / \mathrm{cm}^{2}\right)\right)$ with partial sonophore coverage $\left(f_{\mathrm{BLS}}=0.75, a_{\mathrm{BLS}}=32 \mathrm{~nm}\right)$. Simulations are performed within the framework of the NICE-model (a-d) and the SONIC-model (e-h).(a,h) Membrane charge (red) and voltage (blue) trace. Zoom into the voltage oscillations is visible in the inset for the NICE-model. (b-c,e-f) traces of the gate parameters $(m, n, h$, and $p)) ;(\mathrm{d}, \mathrm{g})$ membrane capacitance plots. 


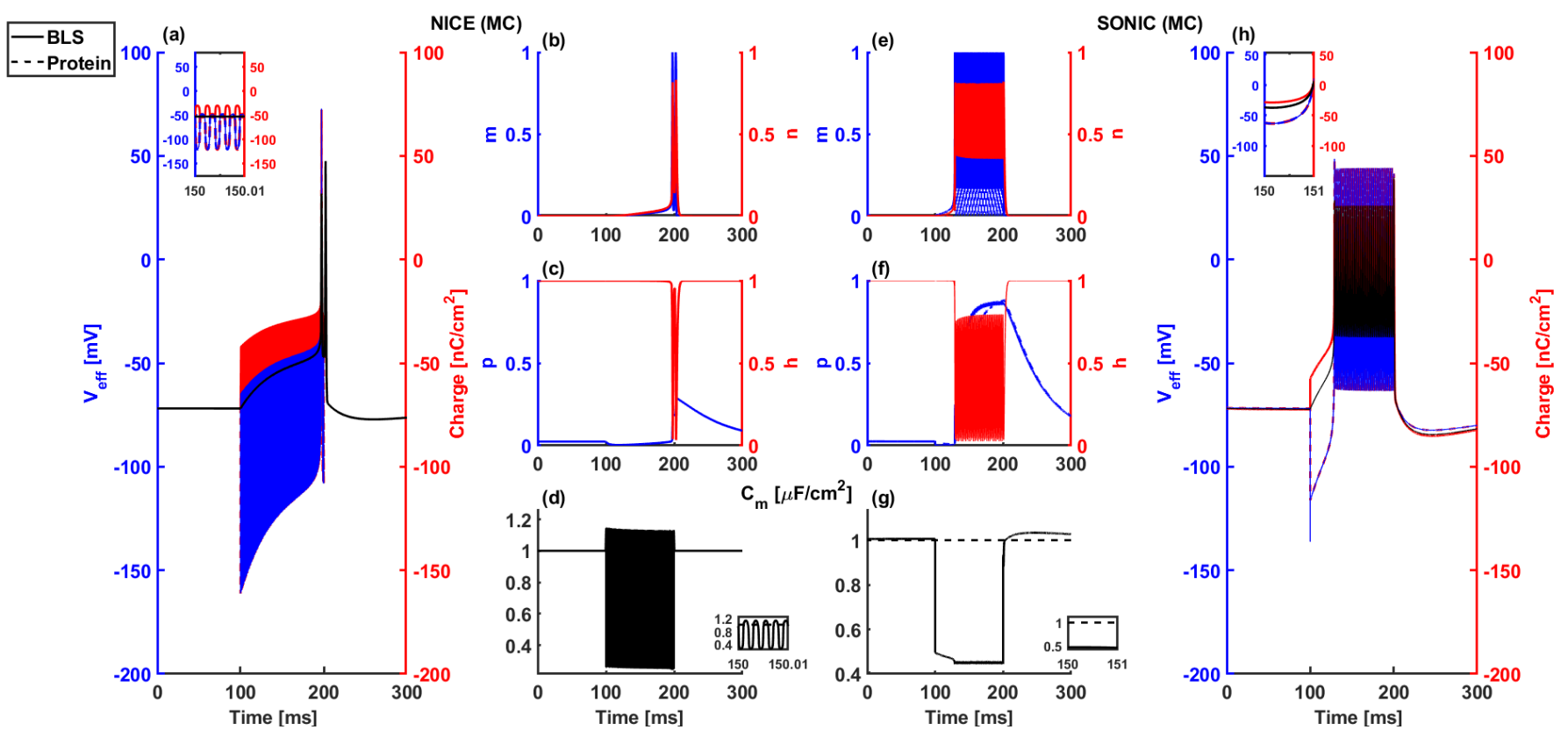

Fig. 2: Insonication of regular spiking neuron (continuous wave ultrasound, pulse onset and pulse duration set to $100 \mathrm{~ms}$, $\left.P_{\mathrm{US}}=100 \mathrm{kPa}\left(I_{\mathrm{sp}}=321 \mathrm{~mW} / \mathrm{cm}^{2}\right)\right)$ with partial sonophore coverage $\left(f_{\mathrm{BLS}}=0.75, a_{\mathrm{BLS}}=32 \mathrm{~nm}\right)$. Two compartments are used to model the bilayer sonophore (full lines) and the surrounding protein islands (dashed lines). Simulations are performed within the framework of the NICE-model (a-d) and the SONIC-model (e-h). (a,h) Membrane charge (red) and voltage (blue) trace. The total averaged membrane charge per surface area is depicted in black. Zoom into the charge and voltage oscillations is visible in the inset for the NICE-model. (b-c,e-f) traces of the gate parameters $(m, n, h$, and $p)) ;(\mathrm{d}, \mathrm{g})$ membrane capacitance plots.

solver is used due to the increased stiffness caused by the tight resistive connection between both compartments (stiff VSVO solver with order between 1 and 5 based on the numerical differentiation formulas, relative tolerance 0.0001 absolute tolerance $0.1 \mathrm{nV}$ (voltage) and $10^{-7}$ (gates), same time step).

To further reduce the stiffness of the equation set, the charge recasting of the SONIC-implementation discussed in the next subsection ( $(18)$, subsection II-E) is also used for the NICEimplementation, because charge recasting does not imply additional assumptions. Despite the optimizations regarding the introduction of the update time $T_{\text {up }}$ to decouple the mechanical and electrodynamical equation sets, a high level of numerical stiffness is still intrinsic to the NICE-implementation due to the dependency of the maximal time step $\mathrm{d} t$ on the ultrasonic period. Furthermore, the Rayleigh-Plesset equation has to be solved every $T_{\text {up }}$, further slowing down the simulations.

\section{E. Multiscale optimized model of intramembrane cavitation}

The multiscale optimized model of neuronal intramembrane cavitation (SONIC) by Lemaire et al. (2019) [30], introduces optimizations w.r.t. the NICE-implementation, in order to achieve increased computational efficiency (three orders of magnitude decrease in simulation time).

First, the stiffness of the PSK-model is reduced by recasting all equations as function of charge $Q_{\mathrm{m}}=C_{\mathrm{m}} V$, e.g.:

$$
\frac{\mathrm{d} Q_{\mathrm{m}}}{\mathrm{d} t}=C_{\mathrm{m}} \frac{\mathrm{d} V}{\mathrm{~d} t}+V \frac{\mathrm{d} C_{\mathrm{m}}}{\mathrm{d} t} .
$$

For a point neuron, the membrane charge changes on a millisecond timescale, driven by transmembrane currents. In contrast, the capacitance and membrane voltage change on a microsecond scale, determined by the ultrasonic frequency. However, for multi-compartmental neurons strong axial currents are able to decrease the local membrane charge timescale towards the ultrasonic period.

Second, the computationally intensive molecular pressure integral is replaced by a Lennard-Jones fit (non-linear least squares fit of $\left.\tilde{A}_{r}, \tilde{\Delta}^{*}, \tilde{x}, \tilde{y}\right)$ :

$$
\tilde{P}_{\mathrm{M}}=\tilde{A}_{r}\left[\left(\frac{\tilde{\Delta}^{*}}{2 Z+\Delta}\right)^{\tilde{x}}-\left(\frac{\tilde{\Delta}^{*}}{2 Z+\Delta}\right)^{\tilde{y}}\right]
$$

Third, effective rate functions $\alpha_{X}^{*}$ and $\beta_{X}^{*}$ are defined and pretabulated as function of the ultrasonic frequency $f_{\mathrm{US}}=1 / T_{\mathrm{US}}$, intensity $I_{\mathrm{US}}$, membrane charge $Q_{\mathrm{m}}$, bilayer sonophore radius $a_{\mathrm{BLS}}$, and sonophore coverage $f_{\mathrm{BLS}}$ (cfr. Fig. S1):

$\alpha_{X}^{*}=\frac{1}{T_{\mathrm{US}}} \int_{0}^{T_{\mathrm{US}}} \alpha_{X}(V(t)) \mathrm{d} t ; \beta_{X}^{*}=\frac{1}{T_{\mathrm{US}}} \int_{0}^{T_{\mathrm{US}}} \beta_{X}(V(t)) \mathrm{d} t$.

Similar tables are defined for the effective membrane voltage:

$$
V^{*}=\frac{1}{T_{\mathrm{US}}} \int_{0}^{T_{\mathrm{US}}} V(t) \mathrm{d} t .
$$

Here, the capacitance $C_{\mathrm{m}}(t)$ in $V(t)=Q_{\mathrm{m}} / C_{\mathrm{m}}(t)$ is obtained by solving the Rayleigh-Plesset equation by fixed membrane charge until periodicity (determined by thresholding the autocorrelation in our implementation) with an ode113 or ode $23 \mathrm{t}$ 


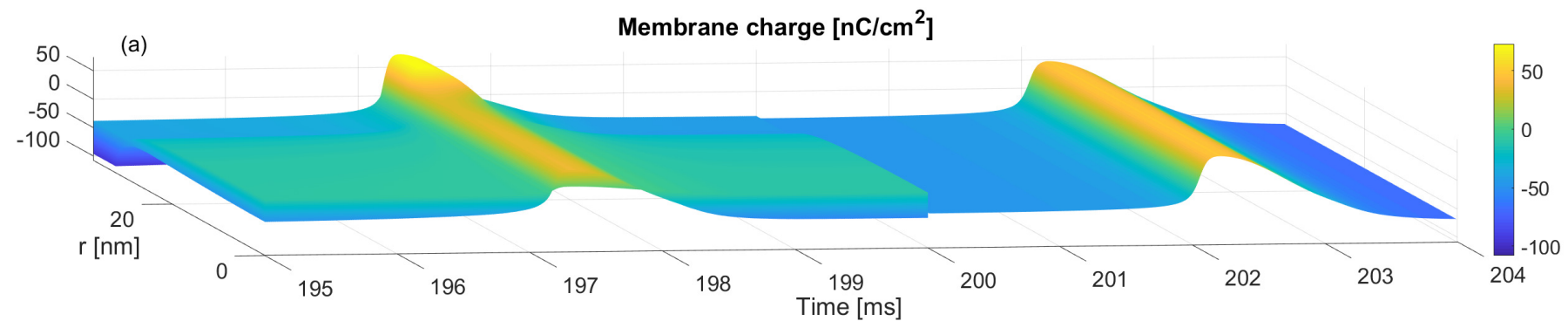

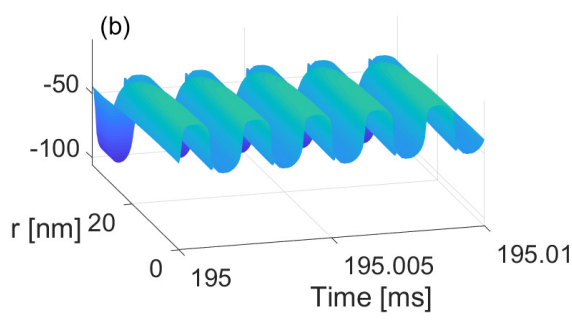

Leaflet displacement [nm]
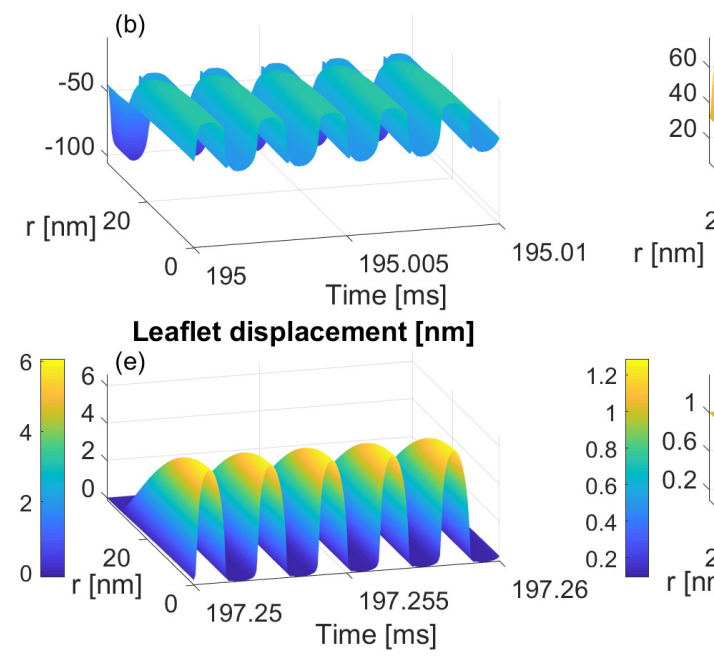

(c)

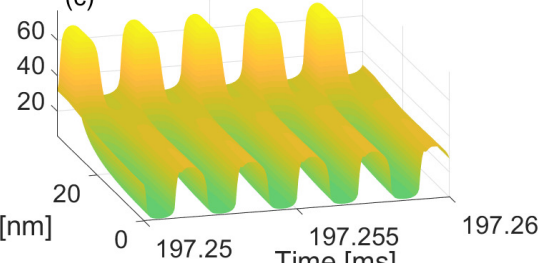

Capacitance $\left[\mu \mathrm{F} / \mathrm{cm}^{2}\right]$

(f)

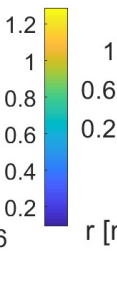

$-25($ d)
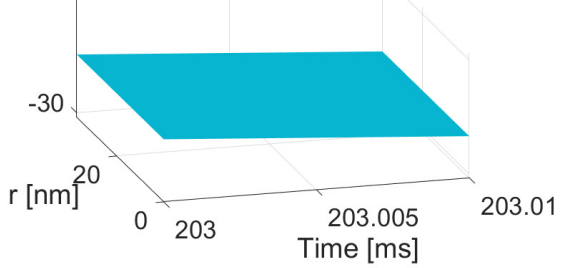

Charge redistribution $[-]$

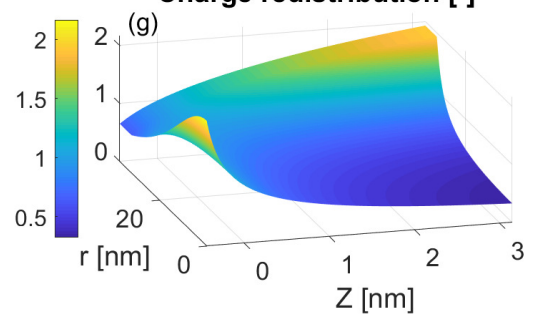

Fig. 3: Illustration of charge redistribution along the bilayer sonophore and protein islands in a point regular spiking model. (a) Membrane charge as function of time and location along the BLS. (b-c) Magnified view of charge oscillations before (b) and during (c) the US-induced action potential. Although total membrane charge does not change on this timescale, the local charge redistributes during leaflet expansion. (d) Magnified view of the membrane charge after US-offset. (e) Local leaflet displacement and (f) capacitance during the action potential. (g) Charge redistribution factor as function of the maximal apex deflection $Z$ and the position along the bilayer sonophore $r$.

(VSVO solver implementing the trapezoidal rule) Matlab ${ }^{\circledR}$ solver (same tolerances and time step as in subsection II-D).

Fourth, the charge-recasted Hodgkin-Huxley and rate equations are integrated by an ode113 (point neuron) or ode15s (multi-compartmental model) solver (same tolerances as in subsection II-D, but with a time step $\mathrm{d} t=50 \mu \mathrm{s}$ :

$$
\begin{aligned}
& \left(\frac{\mathrm{d} Q}{\mathrm{~d} t}\right)^{*}=-\sum_{i} g_{i}\left(V^{*}-E_{i}\right) \\
& \left(\frac{\mathrm{d} X}{\mathrm{~d} t}\right)^{*}=\alpha_{X}^{*}(1-X)-\beta_{X}^{*} X
\end{aligned}
$$

Here, the SONIC-tables $V^{*}, \alpha_{X}^{*}$ and $\beta_{X}^{*}$ are interpolated at runtime.

As in Lemaire et al. [30], the membrane charge is sampled with step $1 \mathrm{nC} / \mathrm{cm}^{2}\left(Q_{\mathrm{m} 0}-25 \mathrm{nC} / \mathrm{cm}^{2}\right.$ to $\left.50 \mathrm{nC} / \mathrm{cm}^{2}\right)$. The ultrasonic intensity table-dimension consists of $0 \mathrm{kPa}$ and 50 logarithmically distributed samples between $30 \mathrm{kPa}$ and $600 \mathrm{kPa}$. The bilayer sonophore coverage $f_{\mathrm{BLS}}$ is sampled with step 0.05 .

\section{RESUltS}

\section{A. Importance of charge redistribution and oscillations}

The simulated neuronal response to ultrasonic insonication in the NICE and SONIC implementation of a point neuron is illustrated in Fig. 1. In the NICE model, the average membrane charge and gate parameters are slowly changing on a millisecond time scale (Fig. 11.a) (red trace) and Fig. 1.bc)). In contrast, the membrane voltage and capacitance are oscillating on the timescale determined by the ultrasonic frequency (Fig. 11 a,d), blue trace).

The most critical assumption within the SONICimplementation is the separation of state variables in two sets, with a fast and slow timescale, respectively (multiscale approximation). Membrane charge and gates should follow a slow millisecond timescale, while displacement and intramembrane gas content act on a fast microsecond timescale. As observed, this assumption is satisfied for a point neuron (Fig. 1 (a-d)). Consequently, the SONICimplementation yields accurate results within significantly shorter computational time (Fig. 1. (e-h) and Fig. 5 in Lemaire et al. (2019) [30]; in [30] a three orders of magnitude gain in algorithmic efficiency for the SONIC-model is reported for a typical continuous wave insonication simulation). Small shifts in excitation thresholds and minor discrepancies of charge traces between the NICE and SONIC point neuron model can be observed in Fig. 1 and are expected due to the multi-scale optimizations [30]. No oscillations are observed in the effective potential $V_{\text {eff }}$ and effective capacitance $C_{\mathrm{m} \text {,eff }}$ in 

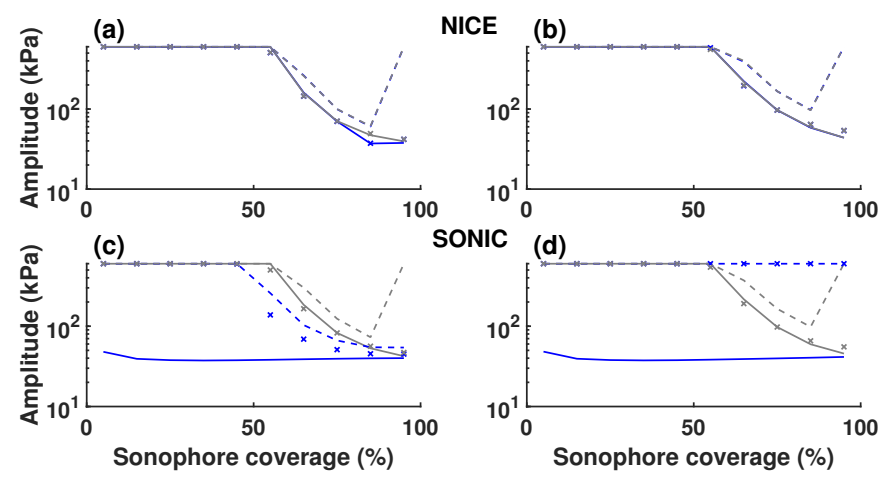

Fig. 4: Excitation thresholds in the NICE (a-b) and SONIC (c-d) model for a regular spiking neuron (continuous wave ultrasound, pulse onset and pulse duration set to $100 \mathrm{~ms}$ ) as function of sonophore coverage $f_{\mathrm{BLS}}(\%)$. Excitation thresholds are determined, while including $(\mathrm{a}, \mathrm{c})$ and excluding $(\mathrm{b}, \mathrm{d})$ action potentials caused by stimulus break after the ultrasonic pulse is turned off. The thresholds for a point-like (gray) and two-compartment model (blue) are compared for full protein coverage (full lines) and partial protein coverage $\left(f_{0}=0\right.$ : dashed, $f_{0}=0.75$ : crosses; $f_{0}$ is defined by the relation between the gain in the protein islands $G_{\mathrm{P}}$ and the measured average gain per surface area $\left.G_{\mathrm{X}}: G_{\mathrm{P}}=\frac{G_{\mathrm{X}}}{1-f_{0}}\right)$. Excitation thresholds that are at the upper titration limit $(600 \mathrm{kPa})$ imply that no excitation is observed for the corresponding sonophore coverage.

the SONIC-model (Fig. 1 $(\mathrm{g}, \mathrm{h})$ ), because these quantities are defined within the SONIC-framework as temporal averages over the ultrasonic period.

Charge redistribution across the bilayer sonophore and protein islands is implicit in the NICE-implementation (illustrated in Fig. 3). During insonication and before the depolarization threshold is reached with subsequent spiking, the total membrane charge is slowly increasing. During this charge build up, the leaflet expansion is accompanied by a drop in local capacitance in the sonophore (Fig. 3 (e-f)). The change in capacitance during intramembrane cavitation expansion is most significant in the middle of the sonophore and drops towards the protein islands. Consequently, charge is redistributed from the bilayer sonophore to the protein islands, since the point neuron description implies a potential spatial gradient equal to zero (Fig. 3 b)). Similarly, at the peak depolarization of the action potential, charge is redistributed from the sonophore to the surrounding proteins during bilayer expansion, but now with opposite sign (Fig. 3(c)). After ultrasonic stimulus offset, the leaflet does not periodically inflate and deflate anymore and charge density is constant along the bilayer sonophore and surrounding protein islands (Fig. 3.d)).

In Fig. $3 \mathrm{~g}$ ) the charge redistrition factor $r_{\mathrm{Q}}=q(r, t) / Q_{\mathrm{m}}$ is depicted as function of the radial position $r$ along the sonophore and proteins $(r=0$ is the center of the sonophore, cfr. Fig. S1) and the maximal apex deflection $Z$. Indeed, for positive deflections $(Z>0)$ charge from the bilayer sonophore is distributed equally over the protein islands. Conversely, during negative deflections $(Z<0)$ the center of the bilayer
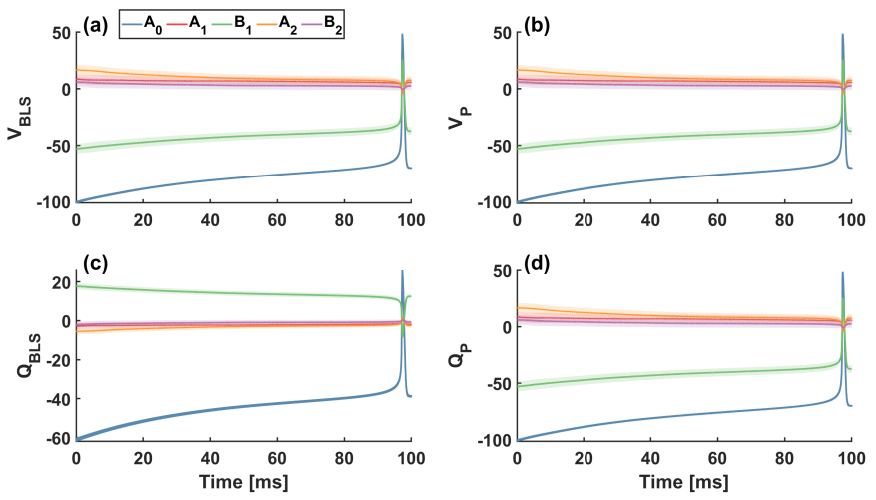

Fig. 5: Temporal dynamics of effective values and first two overtones of membrane potential (a-b) and charge (c-d) at the bilayer sonophore level $(\mathrm{a}, \mathrm{c})$ and the protein islands $(\mathrm{b}, \mathrm{d})$. Shaded areas represent the corresponding $95 \%$ confidence bounds.

sonophore will have the highest charge density values (peak in the bottom-left of Fig. 3 (g)).

The nanoscale two-compartment model results of the NICE and SONIC model are shown in Fig. 2. For the NICE implementation we observe similar results to the single compartment model for the total membrane charge $Q_{\mathrm{m}}$ (Fig. 2 a); black trace), sonophore and protein membrane voltage ( $V_{\mathrm{BLS}}$ and $V_{\mathrm{P}}$. Fig. 2(a); dashed and full blue trace), gate parameters (Figs. 2(b-d)), and capacitance (Fig. 2(d)). Furthermore, $V_{\mathrm{BLS}}(t) \approx V_{\mathrm{P}}(t)$, implying that a single compartment pointneuron model is sufficient for the nanoscale sonophore. This means that we can use the bilayer sonophore and protein islands nanoscale model to benchmark a computationally efficient multi-compartmental model for ultrasonic neuromodulation, because the solution is known by the single compartment model in Fig. 1. As expected from our observations on charge redistribution during leaflet oscillation in Fig. 3, the BLS charge density $Q_{\mathrm{BLS}}$ and protein charge density $Q_{\mathrm{P}}$ show fast (microsecond timescale) anti-phase oscillations (inset Fig. 2 red full and dashed traces).

Conversely, in the nanoscale multi-compartmental SONIC implementation, fast oscillations in the membrane charge are implicitly omitted. In the two-compartment model, this means that the SONIC-model will not take into account the redistribution during the ultrasonic period between the sonophore and the protein islands. Here, we observe that this results in an inaccurate result, as depicted in Fig. 2(e-h). The pseudoperiodic fast charge oscillations (Fig. 3) impact the neuronal response (cfr. NICE-MC and SONIC-MC in Fig. 2) by two non-linear mechanisms. First, because rate functions of the ion channel gates are non-linear (e.g., sigmoidal steady-state gating functions), a periodic charge oscillation will result in altered effective (cycle-averaged) ion channel dynamics. Second, the electrostatic pressure $P_{\mathrm{ec}}$ (6) is proportional to the square of the charge oscillation amplitude. As a result, this oscillation in membrane charge will also influence the effective capacitance and voltage. Note that the very fast change in membrane charge at insonication onset in Fig. 2(h), driven by 
strong axial currents, also breaks the underlying assumption of a slow charge timescale.

\section{B. Excitability: charge oscillations and protein coverage}

The excitability of the point (gray) and nanoscale multicompartmental (blue) NICE and SONIC models is shown in Fig. 4. First, we observe that for the point model the excitation curves predicted by the SONIC-model (simulation time is about 10 minutes on a personal computer (quad core, $3.4 \mathrm{GHz}$ clock rate, 16 GB RAM)) is close to the thresholds predicted by the NICE-implementation (simulation time was about 7 days on a single HPC node (36 processors, $2.3 \mathrm{GHz}$ clock rate)), cfr. the gray lines in Fig. 4(a-b) and Fig. 4(c-d): the maximal error is smaller than $24 \%$ and $7.5 \%$ for excitation thresholds including and excluding action potentials caused by stimulus break, respectively (excitation thresholds greater than the upper titration limit $(600 \mathrm{kPa})$ are excluded $)$.

Intramembrane cavitation models with partial bilayer sonophore coverage have implicitly assumed that the protein conductances $G_{\mathrm{X}}$ are not affected by the coverage fraction $f_{\mathrm{BLS}}$ [28], [30]. As a result, higher $f_{\mathrm{BLS}}$ results unequivocally in lower ultrasonic excitation thresholds in these models. However, in reality a higher coverage with bilayer sonophores, implies a lower coverage with membrane proteins. As neuronal excitation by intramembrane cavitation requires both the bilayer sonophore and protein islands, optimal neuronal response might be at intermediate values of $f_{\mathrm{BLS}}$. Here, accurate modeling of excitation thresholds as function of the sonophore coverage, taking into account the trade-off with protein island coverage, is important in order to predict which neuron types might be responsive to ultrasound by the underlying mechanism of intramembrane cavitation. In Fig. 4, excitation thresholds were also calculated (dashed and crosses), when taking into account that sonophore and active protein coverage of the membrane are mutually exclusive. In the point model, partial sonophore coverage is modeled by altering the channel gains of all active conductances, by $G_{\mathrm{X}}^{*}=\left[\left(1-f_{\mathrm{BLS}}\right) /\left(1-f_{0}\right)\right] G_{\mathrm{X}}$. Here, $G_{\mathrm{X}}^{*}$ is the modified channel gain and $f_{0}$ takes into account the unknown sonophore fraction for which the original channel gains are determined (equal to 0 and 0.75 for dashed and crosses line, respectively). In other words, $f_{0}$ connects the average channel gain $G_{\mathrm{X}}$ with the local gain in the protein islands $G_{\mathrm{P}}=G_{\mathrm{X}} /\left(1-f_{0}\right)$. Taking into account the partial coverage of protein islands with $f_{0}=0$, results in an ideal coverage fraction for excitability at $f_{\mathrm{BLS}}=85 \%$, caused by the mutual exclusivity of BLS and protein coverage.

Second, in the NICE-implementation (Fig. 4(a-b)) nanoscale multi-compartmental excitation thresholds (blue) and point neuron thresholds (gray) coincide, except for small discrepancies at larger sonophore coverage when excitation at insonication break is taken into account (Fig. 4(a)). In contrast, nanoscale multi-compartmental thresholds in the SONICmodel with full coverage of proteins $\left(G_{\mathrm{X}}^{*}=G_{\mathrm{X}}\right)$ are much
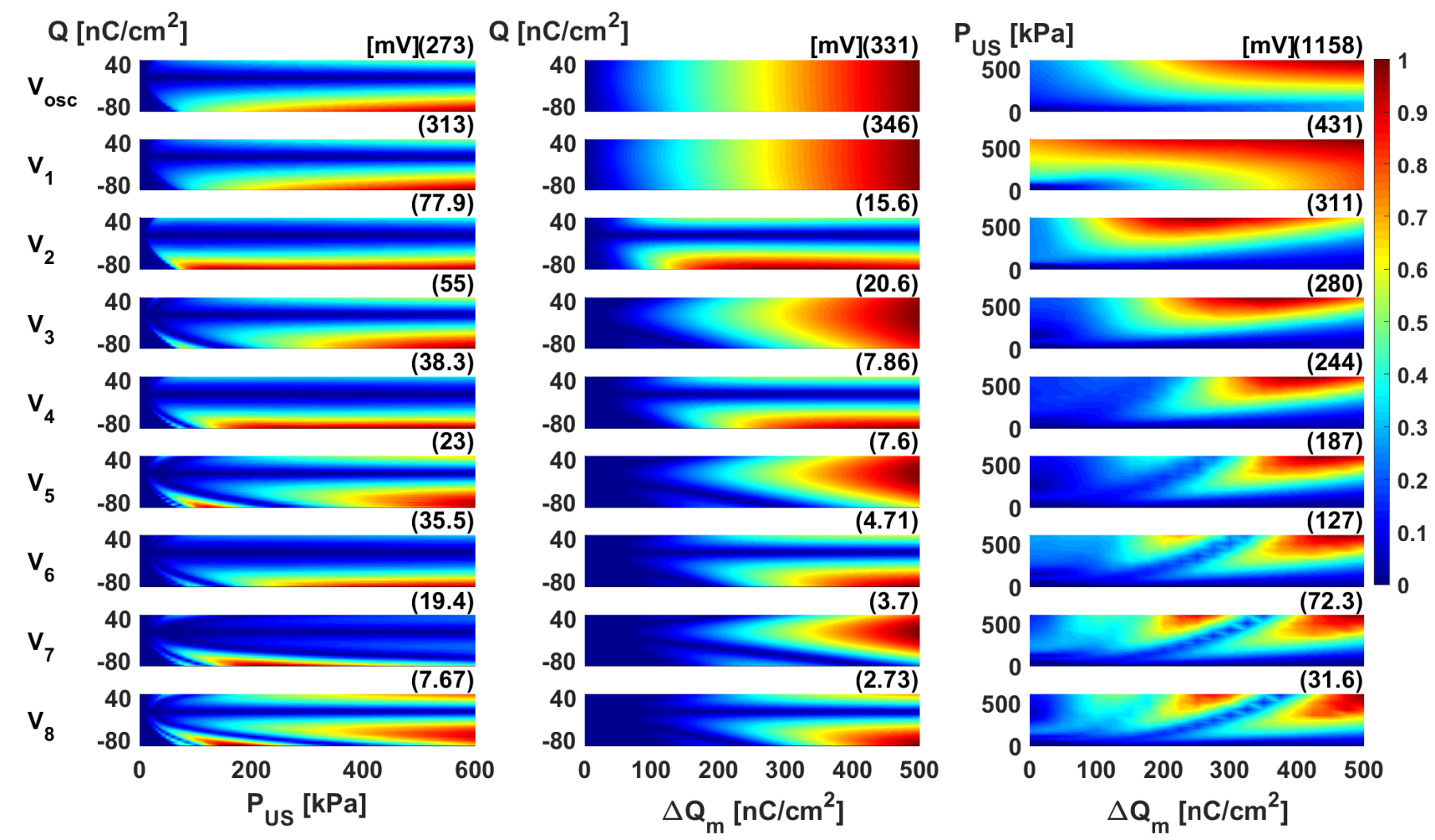

Fig. 6: Normalized amplitude of voltage oscillations $V_{\text {osc }}$ and first eight fourier series amplitudes $V_{i}$ as function of ultrasonic pressure $P_{\mathrm{US}}$, membrane charge $Q$ and membrane charge oscillations $\Delta Q_{m}$. Normalization amplitude is mentioned at the top right of each subplot. 
lower and less sensitive to the sonophore coverage, as observed by Lemaire et al. [30]. However, when partial coverage of proteins is taken into account, the nanoscale multi-compartmental SONIC-model is not excitable (dashed and cross lines in Fig. 4(d)), except by stimulus break (Fig. 4.c)).

\section{Slow dynamics of charge oscillation and impact on voltage oscillations}

We investigate the dynamics of the charge oscillations and their impact on the resulting voltage oscillations, by means of their fourier series. E.g., for the membrane charge: $Q(t)=A_{\mathrm{Q}, 0}+\sum_{k=1}^{N_{\mathrm{FS}}}\left[Q_{k} \cos \left(k \omega t+\phi_{k}\right)\right]=A_{\mathrm{Q}, 0}+$ $\sum_{k=1}^{N_{\mathrm{FS}}}\left[A_{\mathrm{Q}, \mathrm{k}} \cos (k \omega t)+B_{\mathrm{Q}, \mathrm{k}} \sin (k \omega t)\right] \quad\left(N_{\mathrm{FS}}\right.$ : number of fourier series overtones).

The dynamics of the fourier components of the charge and membrane voltage $A_{i}$ and $B_{i}$ are illustrated in Fig. 5 . Here we observe that the fourier components are changing slowly on a millisecond scale, without fast oscillation. Furthermore, the amplitude of the voltage oscillation $V_{\text {osc }}$, the harmonic frequency amplitude $V_{1}$ and the first seven overtone amplitudes $V_{i}(i=2.8)$ are depicted in Fig. 6 as function of the ultrasonic pressure, membrane charge and charge oscillation amplitude. Membrane oscillation components tend to decrease with increasing overtone number. Furthermore, voltage oscillations are stronger for larger charge oscillations or ultrasonic intensities. Also, it is interesting to note that all even fourier components vanish at $Q=0 \mathrm{nC} / \mathrm{cm}^{2}$ and $P_{\mathrm{US}}=0$, due to the symmetry of the electrostatic pressure. We conclude that although charge and voltage have a short microsecond timescale in a multi-compartment model, the multiscale approach of [30] can be extended, by considering the fourier series components of the oscillation instead. Indeed, these fourier components are changing on a slow timescale and their effect on the voltage oscillations implies that the multi-compartmental UNMOD-model has to account for only a limited number of components.

\section{Discussion}

In this study, the importance of charge oscillations and redistribution in multi-compartmental implementations of the PSKUNMOD model is demonstrated. To this end, the nanoscale model of the bilayer sonophore and surrounding protein islands is chosen as a benchmark. As the nanoscale model is close to equipotential, single compartment solutions are accurate and can be used to validate multi-compartmental results (cfr. Fig. 1 (a-d) and Fig. 2(a-d)). Here, it is important to observe that the studied nanoscale BLS-Protein structure is in fact a spatially averaged model, because we do not account for the stochastic currents of individual voltage-gated channels. Instead, transmembrane currents are modeled by a macroscopic Hodgkin-Huxley framework. This approach is valid, because ion channels charge the membrane on a millisecond timescale. Consequently, stochasticity of individual protein channels will not affect fast charge oscillations, present on a microsecond timescale. A single nanometric and idealized (Hodgkin-Huxley type) bilayer sonophore and protein structure can then be used to predict the response of a micrometer-scale neuronal compartment, under the assumption that the size of this compartment is much lower than the electrotonic length and the ultrasonic wavelength [27]. The first condition in terms of the electrotonic length implies that all individual stochastic protein channels are connected in parallel between the same intraand extracellular potentials, allowing for the spatial averaging that is necessary for the applicability of the Hodgkin-Huxley model. The second condition on the ultrasonic wavelength ensures that all bilayer sonophores have a similar temporal evolution, required for spatial averaging over the individual sonophores within a neuronal compartment. Both conditions are expected to be satisfied for practical ultrasonic frequencies and neuronal compartment dimensions. Furthermore, this discussion implies that the spatial discretization scale in future morphologically realistic ultrasonic neuromodulation models, is completely determined by the electrotonic length and the ultrasonic wavelength. In other words, although ultrasonic stimulation by intramembrane cavitation acts on nanometric sonophores, spatial discretization can be performed on a morphological scale (millimeter order of magnitude), by using spatial averaging in order to include the relative contribution of the sonophores and the protein islands.

The explicit modeling of fast charge redistribution and oscillations is important to obtain accurate excitation thresholds and neuronal responses. Indeed, although the SONIC-model results in accurate predictions for the point-neuron model (cfr. Fig. 11(a-d) and Fig. 1 (e-h)), inaccurate tonic spiking is observed when applying SONIC to the two-compartment nanoscale model (Fig. 2(e-h)). This can be explained by the fact that SONIC will not take into account fast charge oscillations in individual compartments (Fig. 3). The pseudoperiodic fast charge redistribution will influence the excitation thresholds (Fig. 4) by two non-linear mechanisms: the electrostatic interaction term in the Rayleigh-Plesset equation and the rate functions of ion channel gating.

Furthermore, the impact of the mutual exclusivity between sonophore and protein coverage on excitability is investigated, by comparing the excitation thresholds of two partial protein coverage models (i.e., no voltage-gated protein channels in the sonophore compartment, $f_{0}=0$ and $f_{0}=0.75$ ) with a BLSProtein model with full protein coverage. This third model case contains voltage-gated channels within the sonophore compartment and as a result is not intended to be biologically realistic. However, this full protein coverage model is useful in order to compare simulation results with prior literature and in order to quantify the impact on excitability of the mutual exclusivity of protein and sonophore coverage. Furthermore, the full protein coverage model is relevant if the nanoscale bilayer sonophore and protein structure is used as a benchmark and test-model for future computationally efficient multi-compartmental UNMOD models, because spatially-extended neuron models will often have ion channels in connected compartments as well (e.g., subsequent Ranvier nodes in a myelinated fibre model). Although there are significant differences between the three simulated model cases, the importance of charge oscillations in terms of neuronal excitability is observed both for partial and full protein coverage structures (Fig. 4). When protein coverage is taken into account, excitation thresholds will not 
decrease unconditionally with sonophore coverage, but reach a minimum at intermediate coverage fractions (e.g., $f_{\mathrm{BLS}}=$ $85 \%$ for $f_{0}=0$ ). Neuronal sensitivity to ultrasonic neuromodulation by intramembrane cavitation is likely influenced by the expressed sonophore coverage fraction. Here, accurate modeling of excitability as function of sonophore and protein coverage might explain why different neuron types seem to be sensitive to ultrasound by different mechanisms. E.g., while the predictions of the PSK-UNMOD model of intramembrane cavitation correspond well with ultrasound induced EMGresponses in mice [4], ultrasonic modulation of retinal cells has been linked with acoustic radiation pressure [36]-[38]. Here, a tentative explanation would be suboptimal sonophore coverage in retinal cells, resulting in a high excitation threshold for neuromodulation by intramembrane cavitation as compared to excitation by acoustic radiation.

Subsequently, we showed that the fourier components of these oscillations are slowly changing in time (Fig. 5) and that the resulting voltage oscillation components are decreasing in energy with overtone number (Fig. 6). These results are the first steps in the direction of a computationally efficient and morphologically-realistic neuron model of UNMOD by intramembrane cavitation. Based on the observations in this study, we have designed a multi-scale optimized model for multi-compartmental neurons, SECONIC, that includes a system of equations for the first few fourier series components of the membrane voltage and charge [39]. Multi-scale optimization of the multi-compartmental UNMOD model will be indispensible, as the bilayer sonophore model exhibits high numerical stiffness due to the intrinsically different timescales set by the ultrasonic frequency and the membrane channel dynamics. Indeed, in our preliminary study [29], an attempt was made to simulate ultrasonic neuromodulation of morphologicallyrealistic cells [40], [41] by the NICE-implementation. However, only very short insonication durations could be simulated and solver instabilities were observed, excluding the possibility of exploration of the parameter space or extensive model validation without multi-scale optimization. On the other hand, preliminary simulations in [29] motivated the design of this study, because we observed membrane charge oscillations with amplitudes in the range $10-80 \mathrm{nC} / \mathrm{cm}^{2}$ in a morphologically realistic blue-brain project cortical pyramidal cell [40], [41], upon application of fixed capacitance oscillations. Here, we demonstrated that neuronal excitability is significantly altered in the presence of charge oscillations with similar amplitudes in the nanoscale BLS-Protein structure. The methodology of simulating the insonication-induced neuronal response in a morphologically realistic neuron by enforcing a sinusoidal capacitance oscillation, was necessary due to the mentioned computational stiffness of the NICE-model, i.e., in order to ensure solver stability and reasonable simulation times. This approach removes the Rayleigh-Plesset bilayer sonophore dynamics from the model and this approximation results in qualitatively similar predictions in point neurons [28]. However, enforcing a capacitance oscillation is arguably insufficient in the presence of charge oscillations in multi-compartmental models, because fast redistribution of charge between compartments will impact excitability through the dependence of the sonophore dynamics on the quadratic electrostatic pressure. Consequently, in this study, we used a two-compartment model with the full NICE-implementation instead (i.e., including the Rayleigh-Plesset sonophore dynamics and electrostatic interaction), in order to determine the importance of charge redistribution.

As future work, we intend to expand on this study, by performing a systematical analysis in morphologically realistic neurons in order to quantify the relative impact of charge oscillations on the neuronal response as function of the ultrasonic waveform, neuronal morphological and physiological parameters, etc., by comparing the predictions of the multiscale optimized SONIC [30] and SECONIC [39] frameworks. In the context of this study and preceding these more extensive future work simulations, we performed a sensitivity analysis of the effective depth parameter $d_{\text {eff }}$, in order to quantify the impact of the intracellular coupling strength on the charge oscillation amplitude. Here, the effective depth (default value is $100 \mathrm{~nm}$ in this study) is of interest for this parameter sweep, because $d_{\text {eff }}$ is inversely proportional with the coupling resistance between the compartments (cfr. (14)) and because of the uncertainty associated with its value. However, the range of values for the effective depth included in this sensitivity analysis is not intended to be biologically realistic: instead, the aim is to determine theoretically the dependency of charge oscillations and SONIC accuracy on intercompartmental coupling strength. In Fig. S2-S4 in the supplementary information, it is shown for continuous wave insonication $\left(P_{\mathrm{US}}=300 \mathrm{kPa}\right)$ of the regular spiking cell with partial $\left(f_{0}=0.75\right.$ and $\left.f_{0}=0\right)$ and full protein coverage, that the charge oscillation amplitude is proportional with the effective depth. Furthermore, decreasing the effective depth with five orders of magnitude $\left(d_{\mathrm{eff}} \leq 1 \mathrm{pm}\right.$, i.e. $R_{\mathrm{BLS}, \mathrm{P}} \geq$ $122 \mathrm{G} \Omega$ or $\left.G_{\mathrm{BLS}, \mathrm{P}}\left(=R_{\mathrm{BLS}, \mathrm{P}}^{-1}\right) \leq 8.18 \mathrm{pS}\right)$, results in small charge oscillation amplitudes and accurate simulation results with the SONIC model. This dependency of charge oscillation amplitude and SONIC accuracy on the effective depth was obtained both for the partial protein coverage with $f_{0}=0$ and $f_{0}=0.75$, and for full protein coverage BLS-Protein structures, indicating the importance of intercompartmental coupling on the charge oscillation amplitude.

Although our focus is on the bilayer sonophore model of ultrasonic neuromodulation, the underlying mechanism of ultrasonic neuromodulation is not yet understood and alternative models of UNMOD have been proposed, as mentioned above. For instance, mechanosensitive ion channels that alter their dynamics in the presence of membrane stretch have been observed [42]-[47]. As ion channels alter their conformation upon opening and closing, mechanosensitivity of ion channels is indeed expected. While the harmonic pressure component drives membrane expansion and neuronal excitation in the bilayer sonophore model, other models refer to mechanisms via the acoustic radiation force (ARF). For example, simultaneous bending of both leaflets by the ARF alters the membrane capacitance in the Prieto model [48]. Also, in the Petrov model [49], membrane bending is linked with flexoelectric depolarization due to the electric phospholipid dipole moment. Conversely, membrane depolarization will also cause 
deformation of the bilipid layer. Interestingly, a computational study by Chen et al. [50], investigates the role of the ARF via the flexoelectric effect in UNMOD. Finally, thermodynamical models of the action potential (Heimburg-Jackson model) [51] are relevant within the context of ultrasonic neuromodulation, as they describe the action potential in terms of density pulses (solitons). Although no extension of the Heimburg-Jackson model exists that predicts features of ultrasonic neuromodulation, it is interesting to note that the soliton model can predict pressure reversal from anesthesia. Furthermore, models based on the Heimburg-Jackson model exist that incorporate axoplasmic and membrane pressure waves [52]-[54].

In conclusion, although several possible underlying mechanisms of UNMOD have been put forward, it is not yet clear which mechanism is more dominant. Possibly, ultrasonic neuromodulation can only be understood in terms of multiple interacting mechanisms (e.g., membrane stretch caused by inflating bilayer sonophores could drive altered dynamics in mechanosensitive channels). Furthermore, it is likely that the importance of a specific proposed mechanism depends critically on the neuronal morphology, electrodynamics and mechanical properties, as well as on the ultrasonic waveform (pulse repetition frequency, duty cycle, intensity...). Here, we argue that our understanding of the prerequisites in terms of stimulus waveform or neuron electrodynamical properties of a tentative underlying mechanism can be improved by simulation of its predictions in increasingly realistic models.

\section{CONCLUSiON}

We start from the NICE and SONIC implementations of ultrasonic neuromodulation by intramembrane cavitation to demonstrate the importance of membrane charge redistribution for the construction of a computationally efficient multicompartmental UNMOD model. Although SONIC allows for fast and accurate simulation of single-compartment neurons, its construction implies that the membrane charge is changing on a slow timescale, excluding fast charge redistribution. However, our results indicate that these membrane charge oscillations, caused by strong axial intercompartmental currents driven by large ultrasound-induced voltage oscillations, are important in multi-compartmental and morphologicallyrealistic ultrasonic neuromodulation models. Furthermore, inclusion of charge redistribution will alter simulated excitation thresholds. Next, we analyse the importance of accounting for partial protein coverage: excitation thresholds will not be monotonously decreasing with sonophore coverage but reach a minimum at intermediate coverage fractions. Finally, fourier series analysis is used to demonstrate the slow dynamics of the fourier voltage and charge components. Most of the signal energy is contained within the lowest overtone numbers, implying that an extension of the SONIC model is possible by including a limited number of fourier components.

Motivated by the observations presented in this study, we have designed a multi-compartmental computationally efficient multi-scale optimized model SECONIC for ultrasonic neuromodulation by intramembrane cavitation, that includes a set of equations to determine the most important fourier series components, that describe the fast redistribution and oscillation of membrane charge and voltage. As future work, we intend to assess systematically and quantitatively the impact on the neuronal response of charge oscillations in morphologically realistic neurons, by comparing the predictions of the SONIC and SECONIC frameworks.

\section{CONFLICT OF INTERESTS}

The authors declare that there is no conflict of interest regarding the publication of this paper.

\section{REFERENCES}

[1] W. J. Tyler, Y. Tufail, M. Finsterwald, M. L. Tauchmann, E. J. Olson, and C. Majestic, "Remote excitation of neuronal circuits using low-intensity, low-frequency ultrasound," PloS one, vol. 3, no. 10, p. e3511, 2008.

[2] Y. Tufail, A. Matyushov, N. Baldwin, M. L. Tauchmann, J. Georges, A. Yoshihiro, S. I. H. Tillery, and W. J. Tyler, "Transcranial pulsed ultrasound stimulates intact brain circuits," Neuron, vol. 66, no. 5, pp. 681-694, 2010.

[3] W. Legon, T. F. Sato, A. Opitz, J. Mueller, A. Barbour, A. Williams, and W. J. Tyler, "Transcranial focused ultrasound modulates the activity of primary somatosensory cortex in humans," Nature neuroscience, vol. 17, no. 2, p. 322, 2014.

[4] R. L. King, J. R. Brown, W. T. Newsome, and K. B. Pauly, "Effective parameters for ultrasound-induced in vivo neurostimulation," Ultrasound in medicine \& biology, vol. 39, no. 2, pp. 312-331, 2013.

[5] R. L. King, J. R. Brown, and K. B. Pauly, "Localization of ultrasoundinduced in vivo neurostimulation in the mouse model," Ultrasound in medicine \& biology, vol. 40, no. 7, pp. 1512-1522, 2014.

[6] H. Kim, S. J. Taghados, K. Fischer, L.-S. Maeng, S. Park, and S.-S. Yoo, "Noninvasive transcranial stimulation of rat abducens nerve by focused ultrasound," Ultrasound in medicine \& biology, vol. 38, no. 9, pp. $1568-1575,2012$.

[7] H. Kim, A. Chiu, S. D. Lee, K. Fischer, and S.-S. Yoo, "Focused ultrasound-mediated non-invasive brain stimulation: examination of sonication parameters," Brain stimulation, vol. 7, no. 5, pp. 748-756, 2014.

[8] H. Kim, M. Y. Park, S. D. Lee, W. Lee, A. Chiu, and S.-S. Yoo, "Suppression of eeg visual-evoked potentials in rats via neuromodulatory focused ultrasound," Neuroreport, vol. 26, no. 4, p. 211, 2015.

[9] W. Lee, H.-C. Kim, Y. Jung, Y. A. Chung, I.-U. Song, J.-H. Lee, and S.S. Yoo, "Transcranial focused ultrasound stimulation of human primary visual cortex," Scientific reports, vol. 6, p. 34026, 2016.

[10] O. Naor, S. Krupa, and S. Shoham, "Ultrasonic neuromodulation," Journal of neural engineering, vol. 13, no. 3, p. 031003, 2016.

[11] H. Baek, K. J. Pahk, and H. Kim, "A review of low-intensity focused ultrasound for neuromodulation," Biomedical Engineering Letters, pp. $1-8,2017$

[12] L. Ai, P. Bansal, J. K. Mueller, and W. Legon, "Effects of transcranial focused ultrasound on human primary motor cortex using $7 \mathrm{t}$ fmri: a pilot study," BMC neuroscience, vol. 19, no. 1, pp. 1-10, 2018.

[13] K. Yoon, W. Lee, J. E. Lee, L. Xu, P. Croce, L. Foley, and S.-S. Yoo, "Effects of sonication parameters on transcranial focused ultrasound brain stimulation in an ovine model," PloS one, vol. 14, no. 10, 2019.

[14] T. Deffieux, N. Wattiez, M. Tanter, P. Pouget, J.-F. Aubry, and Y. Younan, "Low intensity focused ultrasound modulates monkey visuomotor behavior," Journal of therapeutic ultrasound, vol. 3, no. 1, pp. 1-2, 2015.

[15] J. Kubanek, J. Brown, P. Ye, K. B. Pauly, T. Moore, and W. Newsome, "Transcranial ultrasound selectively biases decision-making in primates," bioRxiv, p. 486134, 2019.

[16] W. Legon, P. Bansal, L. Ai, J. K. Mueller, G. Meekins, and B. Gillick, "Safety of transcranial focused ultrasound for human neuromodulation," bioRxiv, p. 314856, 2018

[17] P. Gaur, K. M. Casey, J. Kubanek, N. Li, M. Mohammadjavadi, Y. Saenz, G. H. Glover, D. M. Bouley, and K. B. Pauly, "Histologic safety of transcranial focused ultrasound neuromodulation and magnetic resonance acoustic radiation force imaging in rhesus macaques and sheep," Brain Stimulation, 2020.

[18] R. Schoeters, T. Tarnaud, W. Joseph, L. Martens, R. Raedt, and E. Tanghe, "Comparison between direct electrical and optogenetic subthalamic nucleus stimulation," in 2018 EMF-Med 1st World Conference on Biomedical Applications of Electromagnetic Fields (EMF-Med). IEEE, 2018, pp. 1-2. 
[19] A. Guru, R. J. Post, Y.-Y. Ho, and M. R. Warden, "Making sense of optogenetics," International Journal of Neuropsychopharmacology, vol. 18, no. 11, p. pyv079, 2015.

[20] E. Pama, L. S. Colzato, and B. Hommel, "Optogenetics as a neuromodulation tool in cognitive neuroscience," Frontiers in psychology, vol. 4, p. 610, 2013.

[21] J. C. Williams, J. Xu, Z. Lu, A. Klimas, X. Chen, C. M. Ambrosi, I. S. Cohen, and E. Entcheva, "Computational optogenetics: empiricallyderived voltage-and light-sensitive channelrhodopsin-2 model," PLoS computational biology, vol. 9, no. 9, p. e1003220, 2013.

[22] R. Chen, G. Romero, M. G. Christiansen, A. Mohr, and P. Anikeeva, "Wireless magnetothermal deep brain stimulation," Science, vol. 347, no. 6229 , pp. 1477-1480, 2015.

[23] W. J. Tyler, "Neuromodulation by focused ultrasound," pp. 1-13, 2019.

[24] I. Laakso, S. Tanaka, S. Koyama, V. De Santis, and A. Hirata, "Intersubject variability in electric fields of motor cortical tdcs," Brain stimulation, vol. 8, no. 5, pp. 906-913, 2015.

[25] M. Mikkonen, I. Laakso, S. Tanaka, and A. Hirata, "Cost of focality in tdcs: Interindividual variability in electric fields," Brain stimulation, vol. 13, no. 1, pp. 117-124, 2020.

[26] B. Krasovitski, V. Frenkel, S. Shoham, and E. Kimmel, "Intramembrane cavitation as a unifying mechanism for ultrasound-induced bioeffects," Proceedings of the National Academy of Sciences, vol. 108, no. 8, pp. 3258-3263, 2011.

[27] M. Plaksin, S. Shoham, and E. Kimmel, "Intramembrane cavitation as a predictive bio-piezoelectric mechanism for ultrasonic brain stimulation," Physical review X, vol. 4, no. 1, p. 011004, 2014.

[28] M. Plaksin, E. Kimmel, and S. Shoham, "Cell-type-selective effects of intramembrane cavitation as a unifying theoretical framework for ultrasonic neuromodulation," eNeuro, vol. 3, no. 3, pp. ENEURO-0136, 2016.

[29] T. Tarnaud, W. Joseph, L. Martens, T. Van Renterghem, and E. Tanghe, "Ultrasonic neuromodulation in multi-compartmental neuron models," 28th Annual Computational Neuroscience Meeting: CNS*2019, vol. 20, no. 56 , p. $161,2019$.

[30] T. Lemaire, E. Neufeld, N. Kuster, and S. Micera, "Understanding ultrasound neuromodulation using a computationally efficient and interpretable model of intramembrane cavitation," Journal of Neural Engineering, 2019.

[31] T. Tarnaud, W. Joseph, L. Martens, and E. Tanghe, "Computational modeling of ultrasonic subthalamic nucleus stimulation," IEEE Transactions on Biomedical Engineering, vol. 66, no. 4, pp. 1155-1164, 2018.

[32] M. Pospischil, M. Toledo-Rodriguez, C. Monier, Z. Piwkowska, T. Bal, Y. Frégnac, H. Markram, and A. Destexhe, "Minimal hodgkin-huxley type models for different classes of cortical and thalamic neurons," Biological cybernetics, vol. 99, no. 4-5, pp. 427-441, 2008.

[33] T. Tarnaud, W. Joseph, L. Martens, T. Van Renterghem, and E. Tanghe, "Interaction of electrical and ultrasonic neuromodulation: a computational study," Brain Stimulation: Basic, Translational, and Clinical Research in Neuromodulation, vol. 12, no. 2, p. 563, 2019.

[34] L. F. Shampine and M. W. Reichelt, "The matlab ode suite," SIAM journal on scientific computing, vol. 18, no. 1, pp. 1-22, 1997.

[35] R. Ashino, M. Nagase, and R. Vaillancourt, "Behind and beyond the matlab ode suite," Computers \& Mathematics with Applications, vol. 40 , no. 4, pp. 491-512, 2000

[36] M. D. Menz, Ö. Oralkan, P. T. Khuri-Yakub, and S. A. Baccus, "Precise neural stimulation in the retina using focused ultrasound," Journal of Neuroscience, vol. 33, no. 10, pp. 4550-4560, 2013.

[37] M. D. Menz, P. Ye, K. Firouzi, K. B. Pauly, B. T. Khuri-Yakub, and S. A. Baccus, "Physical mechanisms of ultrasonic neurostimulation of the retina," bioRxiv, p. 231449, 2017.

[38] M. D. Menz, P. Ye, K. Firouzi, A. Nikoozadeh, K. B. Pauly, P. KhuriYakub, and S. A. Baccus, "Radiation force as a physical mechanism for ultrasonic neurostimulation of the ex vivo retina," Journal of Neuroscience, vol. 39, no. 32, pp. 6251-6264, 2019.

[39] T. Tarnaud, W. Joseph, R. Schoeters, L. Martens, and E. Tanghe, "Seconic: Towards multi-compartmental models for ultrasonic brain stimulation by intramembrane cavitation," Journal of Neural Engineering, vol. 17, no. 5, p. 056010, 2020.

[40] A. S. Aberra, A. V. Peterchev, and W. M. Grill, "Biophysically realistic neuron models for simulation of cortical stimulation," Journal of neural engineering, vol. 15, no. 6, p. 066023, 2018.

[41] H. Markram, E. Muller, S. Ramaswamy, M. W. Reimann, M. Abdellah, C. A. Sanchez, A. Ailamaki, L. Alonso-Nanclares, N. Antille, S. Arsever et al., "Reconstruction and simulation of neocortical microcircuitry," Cell, vol. 163, no. 2, pp. 456-492, 2015.
[42] C. E. Morris and P. F. Juranka, "Nav channel mechanosensitivity: activation and inactivation accelerate reversibly with stretch," Biophysical Journal, vol. 93, no. 3, pp. 822-833, 2007.

[43] F. Sachs, "Stretch-activated ion channels: what are they?" Physiology, vol. 25 , no. 1 , pp. 50-56, 2010.

[44] P. Wiggins and R. Phillips, "Analytic models for mechanotransduction: gating a mechanosensitive channel," Proceedings of the National Academy of Sciences, vol. 101, no. 12, pp. 4071-4076, 2004.

[45] J. Kubanek, J. Shi, J. Marsh, D. Chen, C. Deng, and J. Cui, "Ultrasound modulates ion channel currents," Scientific reports, vol. 6, no. 1, pp. 114, 2016.

[46] M. L. Prieto, K. Firouzi, B. T. Khuri-Yakub, and M. Maduke, "Activation of piezo1 but not nav1. 2 channels by ultrasound at $43 \mathrm{mhz}$," Ultrasound in medicine \& biology, vol. 44, no. 6, pp. 1217-1232, 2018.

[47] P. Jin, L. Y. Jan, and Y.-N. Jan, "Mechanosensitive ion channels: Structural features relevant to mechanotransduction mechanisms," Annual Review of Neuroscience, vol. 43, 2020.

[48] M. L. Prieto, Ö. Oralkan, B. T. Khuri-Yakub, and M. C. Maduke, "Dynamic response of model lipid membranes to ultrasonic radiation force," PLoS One, vol. 8, no. 10, p. e77115, 2013.

[49] A. G. Petrov, "Flexoelectricity of model and living membranes," Biochimica et Biophysica Acta (BBA)-Biomembranes, vol. 1561, no. 1, pp. 1-25, 2002.

[50] H. Chen, D. Garcia-Gonzalez, and A. Jérusalem, "Computational model of the mechanoelectrophysiological coupling in axons with application to neuromodulation," Phys. Rev. E, vol. 99, p. 032406, Mar 2019. [Online]. Available: https://link.aps.org/doi/10.1103/PhysRevE. 99.032406

[51] T. Heimburg and A. D. Jackson, "On soliton propagation in biomembranes and nerves," Proceedings of the National Academy of Sciences of the United States of America, vol. 102, no. 28, pp. 9790-9795, 2005.

[52] J. Engelbrecht, K. Tamm, and T. Peets, "On mathematical modelling of solitary pulses in cylindrical biomembranes," Biomechanics and modeling in mechanobiology, vol. 14, no. 1, pp. 159-167, 2015.

[53] — , "Modelling of processes in nerve fibres at the interface of physiology and mathematics," arXiv preprint arXiv:1906.01261, 2019.

[54] _ "On mechanisms of electromechanophysiological interactions between the components of signals in axons," Proceedings of the Estonian Academy of Sciences, vol. 69, no. 2, pp. 81-96, 2020. 